PROCEEDINGS OF THE

AMERICAN MATHEMATICAL SOCIETY

Volume 133, Number 1, Pages 263-273

S 0002-9939(04)07659-2

Article electronically published on August 24, 2004

\title{
POSITIVELY CURVED SURFACES WITH NO TANGENT SUPPORT PLANE
}

\author{
JOHN MCCUAN
}

(Communicated by Wolfgang Ziller)

\begin{abstract}
We discuss a characterization of positively curved surfaces $M$ with the property that, at each point, the tangent plane to $M$ is not a support plane for the entire surface. Such positively curved surfaces with no tangent support plane necessarily have non-empty boundary, and any portion $B \subset \partial M$ which has convex hull equal to the convex hull of $\partial M$ we call a generating set. This set plays a key role in constructing examples. We give various examples among which there is an embedded topological disk with smallest possible generating set.
\end{abstract}

\section{INTRODUCTION}

Throughout this paper $M$ denotes a smooth positively curved immersion into $\mathbb{R}^{3}$ of a connected surface.

The condition of positive curvature implies that $M$ is strictly locally convex, i.e., the plane $T_{p} M$ tangent to $M$ at $p$ is a support plane for some (intrinsic) neighborhood $N$ of $p$, and $N \cap T_{p} M=\{p\}$. In the case when $M$ is compact and has no boundary, a well-known theorem of Hadamard [4 asserts that $M$ is an ovaloidthe boundary of a (strictly) convex region in $\mathbb{R}^{3}$. Various authors have generalized Hadamard's theorem. We mention in particular that do Carmo and Lima [2] draw essentially the same conclusion for complete surfaces without boundary, though in this case the region in $\mathbb{R}^{3}$ may be non-compact. In either case, every tangent plane is a global support plane for the surface.

M. Ghomi [3] extended Hadamard's theorem to the case when $\partial M \neq \varnothing$. He asked the question:

When does a positively curved surface with boundary lie in the boundary of a strictly convex region?

Ghomi, in fact, asked (and answered) the more general question: When does a submanifold of any dimension lie in the boundary of a strictly convex region? In the case of surfaces in $\mathbb{R}^{3}$ that interests us, Ghomi's result reads as follows.

Received by the editors March 15, 2002.

2000 Mathematics Subject Classification. Primary 53A05.

Parts of this work were carried out with funding from the National Science Foundation at the University of California, Berkeley, the Mathematical Sciences Research Institute, and Georgia Institute of Technology.

(C)2004 American Mathematical Society Reverts to public domain 28 years from publication 


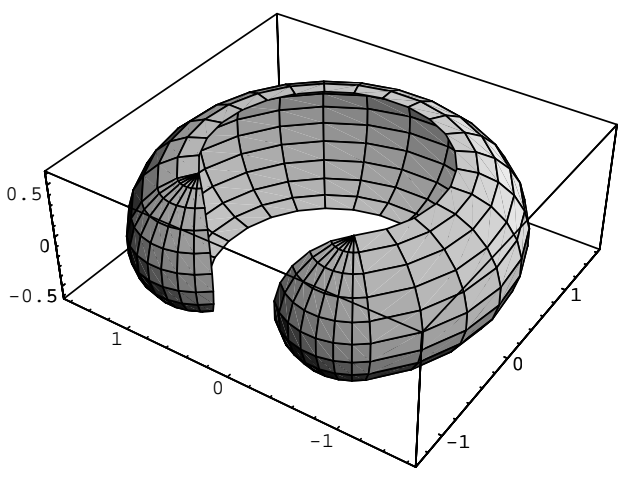

Figure 1. Positively curved surface with boundary.

Theorem (Ghomi [3]). A necessary and sufficient condition that a compact, positively curved surface $M$ is a subset of the boundary of a convex region in $\mathbb{R}^{3}$ is:

For each $p \in \partial M$ the tangent plane $T_{p} M$ is a support plane for $\partial M$, and $\partial M \cap T_{p} M=\{p\}$.

Ghomi notes that his main interest was in addressing a question of Yau [5] Problem 26] which asks:

What characterizes the possible boundary curves of a positively curved embedded disk?

As noted by Ghomi, there are other positively curved embedded disks that are not subsurfaces of ovaloids; see Figure 11 Ghomi and S. Alexander later characterized the larger class of positively curved surfaces with convex boundary, which includes the surface in Figure 1 The boundary of $M$ is said to be convex if $\partial M \subset \partial K$ where $K=K(\partial M)$ denotes the convex hull of $\partial M$. They obtain the following result for embedded surfaces:

Theorem (Alexander-Ghomi [1]). $\partial M$ is convex if and only if

$$
\operatorname{int} M \subset \mathbb{R}^{3} \backslash K \text {. }
$$

When $\partial M$ is convex, one sees from (1) that there is a point $p \in \operatorname{int} M$ with $\operatorname{dist}(p, K)=\max _{q} \operatorname{dist}(q, K)$. It follows that $T_{p} M$ is a global support plane for $M$. Ghomi conjectured, furthermore, that while convex boundary may not characterize the boundary of a positively curved embedded disk, the existence of at least one global support plane should be a necessary condition.

It is interesting that counterexamples, that is, positively curved surfaces $M$ with no tangent support plane, satisfy a characterization that is dual to (11):

Lemma 1. A positively curved immersion $M$ has no tangent support plane if and only if

$$
\operatorname{int} M \subset \operatorname{int} K
$$

and

$$
T_{p} M \cap \operatorname{int} K \neq \varnothing \text { for all } p \in \partial M \text {. }
$$

Proof. It is clear that conditions (2) and (3) imply that there is no tangent support plane. 
Conversely, if there is no tangent support plane, then (2) must hold, or else there is some $p \in \operatorname{int} M$ with $\operatorname{dist}(p, K)=\max _{q} \operatorname{dist}(q, K)$ as in the case of convex boundary.

Finally, if $p \in \partial M$ and $T_{p} M \cap \operatorname{int} K=\varnothing$, then $T_{p} M$ is a support plane for the convex set $K$ and hence for $M$ by (2). This establishes the necessity of (3).

More generally, positively curved surfaces with no tangent support plane are characterized by the condition that for some $A \subset \operatorname{int} M$,

$$
A \subset \operatorname{int} K \text { and } T_{p} M \cap \operatorname{int} K \neq \varnothing \text { for all } p \in M \backslash A .
$$

From this observation we obtain the following lemma.

Lemma 2. If $M$ is a positively curved immersion with no tangent support plane, and $\tilde{M} \subset M$ is a surface with boundary such that the convex hull $\tilde{K}$ of $\partial \tilde{M}$ is $K$, then $\tilde{M}$ has no tangent support plane. In particular, if $B \subset \partial M$ has convex hull $K$ and $B \subset \tilde{M}$, then the conclusion holds.

We call any such set $B \subset \partial M$ with convex hull $K$ a generating set.

Since the convex hull $K$ of $\partial M$ must contain the interior of $M$, the set $K$ must, itself, have nonempty interior. Any generating set $B$ must therefore contain at least four points (not all of which lie on the same plane). Having a finite generating set necessarily requires a surface to have non-smooth boundary. (This follows from the impossibility of a smooth curve lying in, and passing through the vertex of, an acute polyhedral cone.) The examples given below with finite generating sets are subsurfaces of examples with smooth boundary, and as such have a well-defined tangent plane at each boundary point. In this regard we use the following observation.

Lemma 3. If $M$ is a positively curved immersion with no tangent support plane and smooth boundary, and $\tilde{B}$ is a finite subset of $\partial M$ with convex hull a solid polyhedron $\tilde{K}$ each of whose faces is transverse to $M$ along $M \cap \partial K$, then there is a positively curved subsurface $\tilde{M}$ with no tangent support plane and generating set $\tilde{B}$.

Proof. The polyhedron $\tilde{K}$ is an intersection of acute solid polyhedral angles $C_{1}, \ldots$, $C_{k}$ with vertices at the points $q_{1}, \ldots, q_{k}$ of $\tilde{B}$. Reducing slightly each of the angles between the planes at $q_{j}$, one obtains polyhedral angles $\tilde{C}_{1}, \ldots, \tilde{C}_{k}$ whose intersection $\tilde{C}$ lies in int $\tilde{K}$, except for the points $q_{1}, \ldots, q_{k}$. It follows from the transversality assumption that $\tilde{M}=M \cap \tilde{C}$ has piecewise smooth boundary with $\tilde{B}$ as a generating set. Furthermore, $T_{q_{j}} \tilde{M} \cap \operatorname{int} \tilde{K} \neq \varnothing$ for all $j$. It thus follows from Lemma 2 that $\tilde{M}$ has no tangent support plane.

\section{EXAMPLES}

Our examples our constructed explicitly using positively curved strips. A strip (or ribbon) $\mathcal{S}$ is a surface that can be parameterized on a domain $\Sigma=\{(s, t)$ : $\left.T^{-}(s)<t<T^{+}(s)\right\}$ by

$$
X(s, t)=\gamma(s)+f(t) n(s)+t b(s)
$$

where $\gamma: \mathbb{R} \rightarrow \mathbb{R}^{3}$ parameterizes a curve and $f$ is a real-valued function of $t$. The vectors $n$ and $b$ are the normal and binormal of $\gamma$. See Figure 2(a) for an example of a typical domain $\Sigma$. We will assume the curvature $\kappa$ of $\gamma$ satisfies $\kappa=\kappa(s)=|\ddot{\gamma}|>0$. 


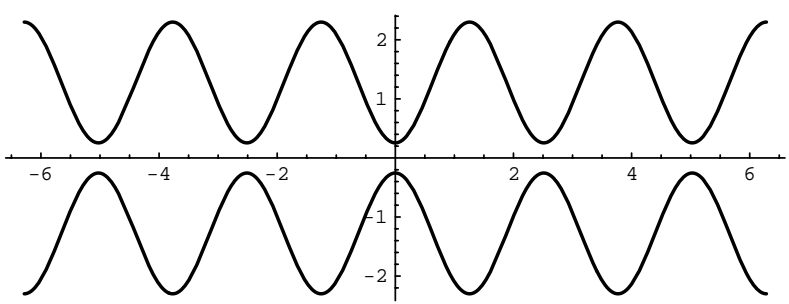

(a)

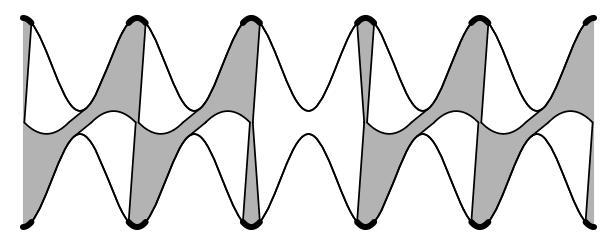

(b)

FiguRE 2. Strip between two periodic curves.

The explicit expression for the Gauss curvature of a strip is somewhat complicated, but the following calculations are adequate for our purposes.

Lemma 4. If the torsion $\tau$ of $\gamma$ is identically 0 (i.e., $\gamma$ is a planar curve), then

$$
K=\frac{f^{\prime \prime} \kappa}{\left(1+f^{\prime 2}\right)^{2}(1-f \kappa)}
$$

For an arbitrary curve $\gamma$, the Gauss curvature of a strip $\mathcal{S}$ along $\gamma($ i.e., for $t=0$ ) is given by

$$
\left.K\right|_{t=0}=\frac{f^{\prime \prime}(0)}{\left(1+f^{\prime}(0)^{2}\right)^{2}} \kappa-\tau^{2} .
$$

These calculations lead immediately to the following corollaries.

Corollary 1. If $\gamma$ is a plane curve and $f$ is a smooth function with $f(0)=0$ and $f^{\prime \prime}>0$ and $f \circ T^{+}(s), f \circ T^{-}(s)<1 / \kappa(s)$ for all $s$ in some interval, then on this interval the strip parameterized by (5) is positively curved.

Corollary 2. Any compact portion of a curve with non-vanishing curvature supports a positively curved strip.

Proof. If $f^{\prime \prime}(0)$ is large enough, the expression (7) will be bounded away from zero. The result follows by continuity.

We first apply Corollary 1 with $\gamma$ given by the "spirograph curve"

$$
\gamma(\theta)=(3 \cos \theta+\rho \cos (3 \theta / 2), 3 \sin \theta-\rho \sin (3 \theta / 2))
$$

determined by a point rigidly fixed at a distance $\rho$ from the center of a circle of radius 2 which is rolling inside a circle of radius 5; see Figure 3] a). We assume initially that $2<\rho \leq 5 / 2$. An elementary calculation then shows

$$
\kappa(\theta)=\frac{3 \rho^{2}-8-2 \rho \cos (5 \theta / 2)}{3\left(\rho^{2}+4-4 \rho \cos (5 \theta / 2)\right)^{3 / 2}}>0 .
$$




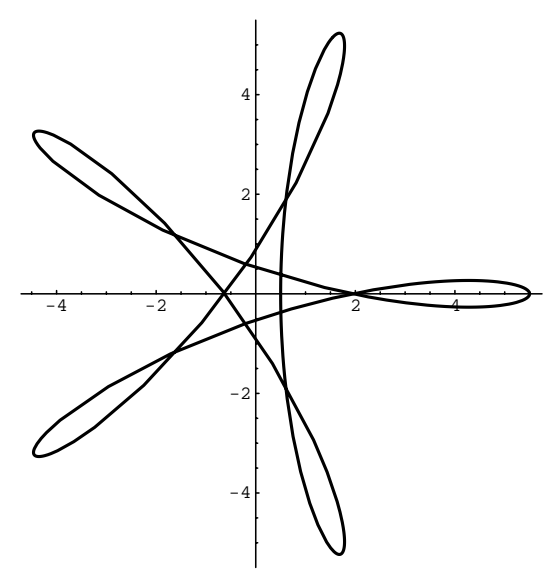

(a)

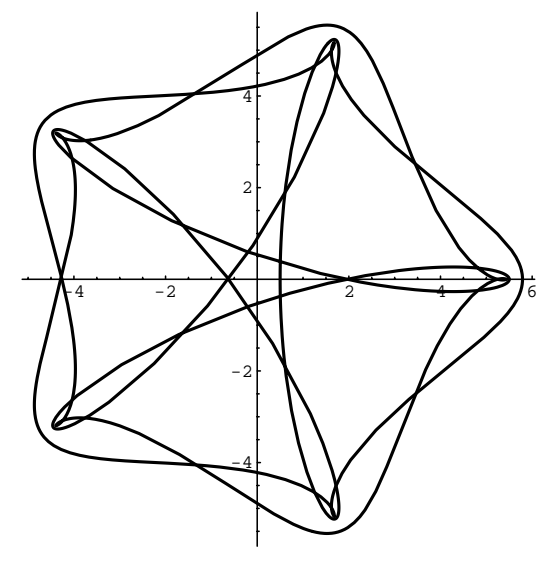

(b)

FiguRE 3. Spirograph curve and the projection of $\partial M$.

Let $T^{ \pm}(\theta)= \pm[A+\epsilon-(A-\epsilon) \cos (5 \theta / 2)]$ where $0<\epsilon \leq 1 / 2 \sqrt{\kappa(0)}<A \leq$ $1 / 2 \sqrt{\kappa(2 \pi / 5)}$. This choice defines a domain $\Sigma$ as in Figure 2(a).

Finally, we take $f(t)=t^{2}$, and taking account of periodicity in Lemma 2 we obtain an annular immersion parameterized by

$$
X(\theta, t)=\gamma+t^{2} n+t e_{3} .
$$

The resulting surface $M$ is composed of five (positively curved) taco shells (see Figure 5). Unlike standard flat taco shells, these curl at the corners. Because of this, they may be smoothly concatenated so that their backs lie successively along the spirograph curve, and their wings are directed outward. The projection into the $x, y$-plane of $\partial M$ is shown in Figure 3(b), and a closeup of the corners where two taco shells join is shown in Figure 4 (a).

The convex hull $K$ of $\partial M$ is generated by ten arcs on the extreme edges of the wings. These arcs are quite small when $A$ is large (say $\geq 2$ ), though Figure 5 corresponds to a considerably lower value of $A$. The fact that the arcs are small can be used to give a proof that $M$ has no tangent support plane as follows.

The largest $z$-values (in magnitude) are obtained when $\kappa$ is at its minima and $t=T^{ \pm}(\theta)$. These boundary values are found to be at $\theta_{k}=2 \pi / 5+4 \pi k / 5$ for $k \in \mathbb{Z}$ and are given by the ten points

$$
X\left(\theta_{k}, T^{ \pm}\left(\theta_{k}\right)\right)=\gamma\left(\theta_{k}\right)+T^{+}\left(\theta_{k}\right)^{2} n \pm T^{+}\left(\theta_{k}\right) e_{3}, \quad k=0, \pm 1, \pm 2 .
$$

The convex hull $K_{0}$ of $\left\{X\left(\theta_{k}, T^{ \pm}\left(\theta_{k}\right)\right)\right\}$ is a pentagonal prism, and $K_{0} \subset K$. One can verify directly that, for large enough values of $A$, most interior points of $M$ are in int $K_{0}$. More precisely, if $A \geq 2$, then the condition $\left|\theta-\theta_{k}\right|>\pi / 8$ for $k=0, \pm 1, \pm 2$ implies

$$
\begin{aligned}
\left|\gamma(\theta)+t^{2} n\right| & \leq|\gamma(\theta)|+T^{+}(\theta)^{2} \\
& <r_{0} \equiv\left(3-\rho+4 A^{2}\right) \cos (\pi / 5) .
\end{aligned}
$$



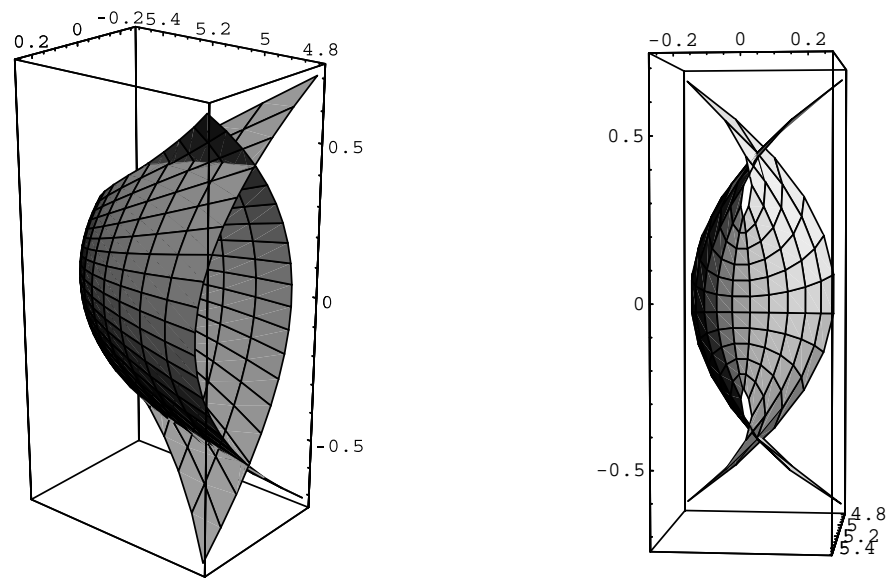

(a)

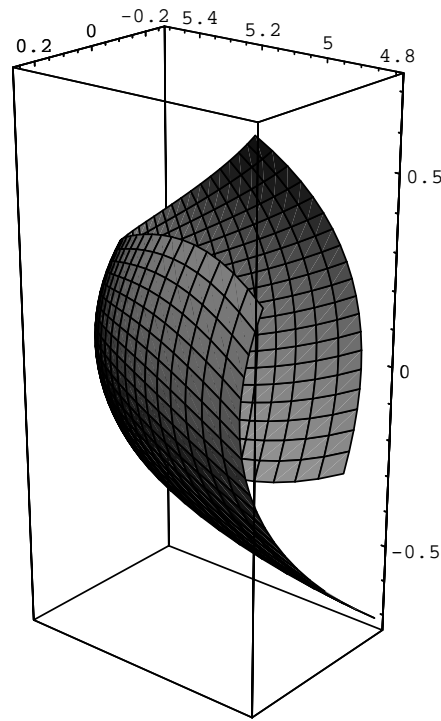

(b)

Figure 4. (a) Where two tacos meet $(-\pi / 8 \leq \theta \leq \pi / 8)$. (b) The desingularization.

The number $r_{0}$ in this inequality is the radius of the circular cylinder inscribed in $K_{0}$. Thus, $X(\theta, t) \in \operatorname{int} K_{0}$.

For the remaining points $p=X(\theta, t)=\gamma+t^{2} n+t e_{3}$ with $\left|\theta-\theta_{k}\right| \leq \pi / 8$ for some $k=0, \pm 1, \pm 2$, we show that $T_{p} M \cap \operatorname{int} K_{0} \neq \varnothing$. The line through $p$ with tangent direction $X_{t}=2 t n+e_{3}$ comes closest to the origin at the point

$$
q=\gamma-t^{2}\left(\frac{1+4 \gamma \cdot n}{4 t^{2}+1}\right) n+2 t\left(\frac{t^{2}-\gamma \cdot n}{4 t^{2}+1}\right) e_{3} \in T_{p} M .
$$

If we specialize to $\rho=5 / 2$, then it follows that $0 \leq \gamma \cdot n \leq 1 / 2$ and, hence, that the third component of $q$ is in the open interval $\left(T^{-}(\theta), T^{+}(\theta)\right)$. Consequently, we 

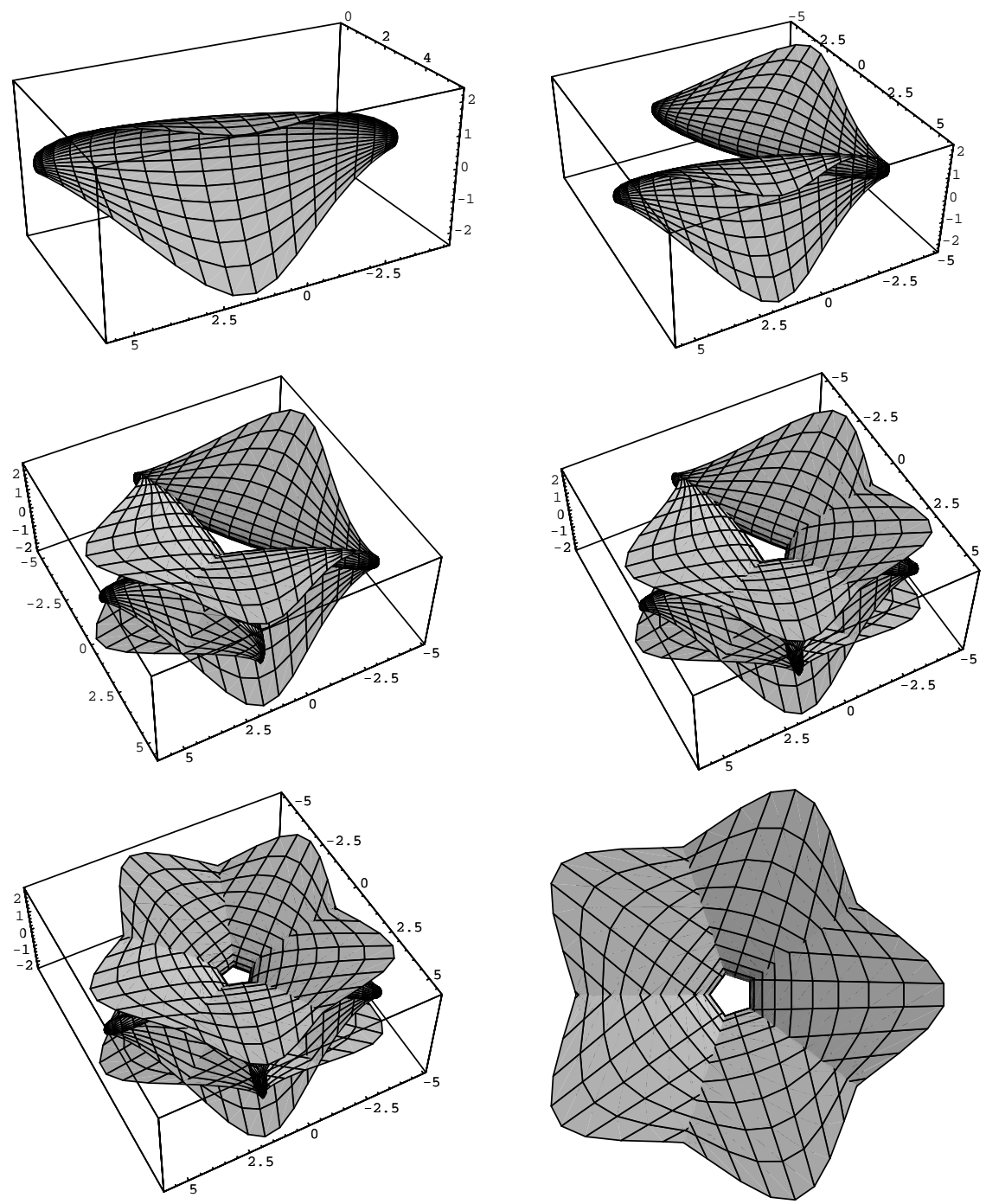

FiguRE 5. Construction of the immersed annular example.

only need to check that the projection

$$
q_{0}=\gamma-t^{2}\left(\frac{1+4 \gamma \cdot n}{4 t^{2}+1}\right) n
$$

lies in the pentagonal base of $K_{0}$. It is easily checked under our present assumptions, $\rho=5 / 2, A \geq 2$, and $\left|\theta-\theta_{k}\right| \leq \pi / 8$, that $|\gamma| \leq 3$. From this it is easy to see that $\left|q_{0}\right|<r_{0}$ where $r_{0}$ is given in (8). Thus, $q \in T_{p} M \cap \operatorname{int} K_{0}$.

By the characterization (44), we conclude that $M$ has no tangent support plane.

By removing portions of $\Sigma$ as indicated in Figure 2(b), we obtain an embedded disk which, since the dark curves correspond to a set $B$ whose convex hull is $K$, has no tangent support plane by Lemma 2. Two views of this surface are shown in 

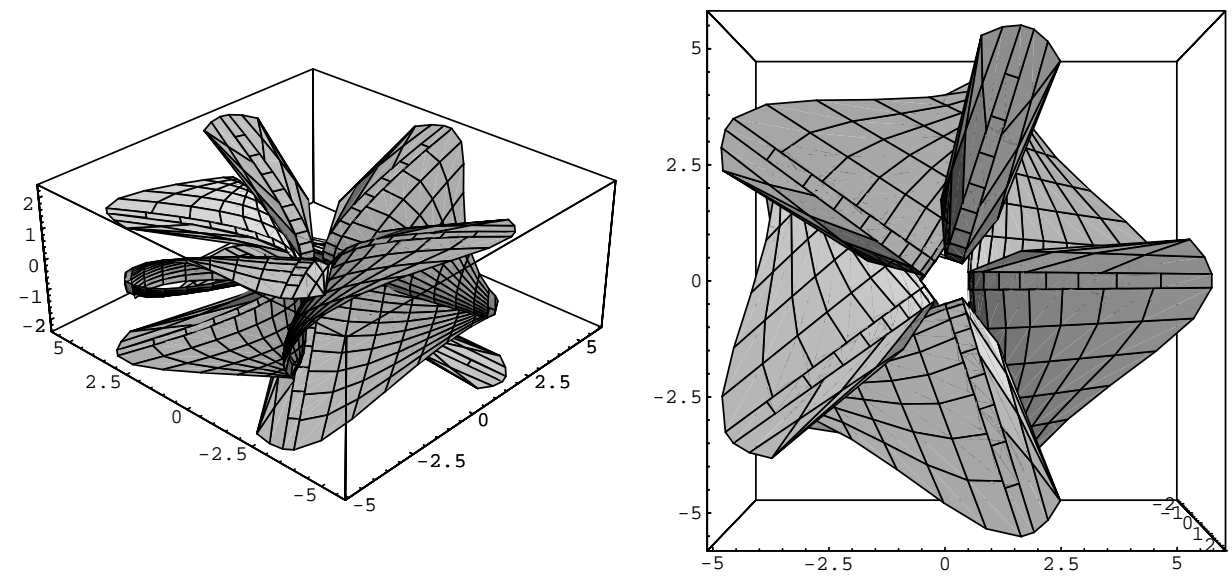

FiguRE 6. An embedded topological disk.

Figure 6. The conditions of Lemma 3 are also easily checked for this surface with $\tilde{B}$ consisting of the ten points $X\left(\theta_{k}, T^{ \pm}\left(\theta_{k}\right)\right), k=0, \pm 1, \pm 2$.

In order to construct an example with four point generating set, we consider the possibility of two strips crossing (and coinciding) in the neighborhood of a point. It is interesting that, at least under simple circumstances, there is only one way for this to occur.

Lemma 5. Let $X_{1}=\gamma_{1}+f_{1} n_{1}+t b_{1}$ and $X_{2}=\gamma_{2}+f_{2} n_{2}+t b_{2}$ be two strips based on plane curves $\gamma_{1}$ and $\gamma_{2}$. If $f_{j}(0)=0=f_{j}^{\prime}(0), j=1,2, \gamma_{1}$ meets $\gamma_{2}$ orthogonally at $p$, and the two strips coincide in a neighborhood of $p$, then $\operatorname{graph}\left(f_{1}\right)$ and $\operatorname{graph}\left(f_{2}\right)$ are portions of the same circular arc. That is, the strips are locally spherical near an intersection point $p$ of the two curves $\gamma_{1}$ and $\gamma_{2}$.

Proof. Without loss of generality, $p=\gamma_{j}(0), j=1,2$.

For each $t$ small enough, $\alpha(s)=\gamma_{1}(s)+f_{1}(t) n_{1}(s)+t b_{1}(s)$ and $\beta(u)=\gamma_{1}\left(s_{0}\right)+$ $f_{2}(u) n_{2}\left(s_{0}\right)+u b_{2}\left(s_{0}\right)$ (where $s_{0}=s_{0}(t)$ is determined by $\gamma_{2}\left(s_{0}\right)=\gamma_{1}(0)+f_{1}(t) n_{1}(0)+$ $\left.t b_{1}(0)\right)$ are tangent curves in the common portion of the strips.

Equating the normal curvatures of these curves at $s=u=0$, one obtains

$$
1+{f_{1}^{\prime}}^{2}=1\left(1-f_{2}^{\prime \prime}(0) f_{1}^{\prime}\right)^{2} .
$$

The result then follows from the uniqueness theorem for ODEs.

The next example, accordingly, will be composed of two strips that cross (and coincide) in a small spherical cap. The strips then branch off to the four vertices of a tetrahedral solid $T$ according to the schematic shown in Figure 7 . The vertices of $T$ are the points $(0, \pm 1,1)$ and $( \pm 1,0,-1)$. The small sphere at the origin represents the small spherical cap $u(x, y)=-r+\sqrt{r^{2}-\left(x^{2}+y^{2}\right)}$ where the two strips cross. The strips, and the surface, are symmetric with respect to the $z$-axis; half of the base curve $\gamma$ for each strip is indicated. The curve $\gamma_{1}$ connecting the origin to $(0,1,1)$ has tangent vector $(1,1,0)$ at the origin and spirals three-quarters of a turn about the positive $y$-axis. The second curve $\gamma_{2}$ has tangent $(1,-1,0)$ at the origin and spirals one-quarter turn about the $x$-axis to reach $(1,0,-1)$. 


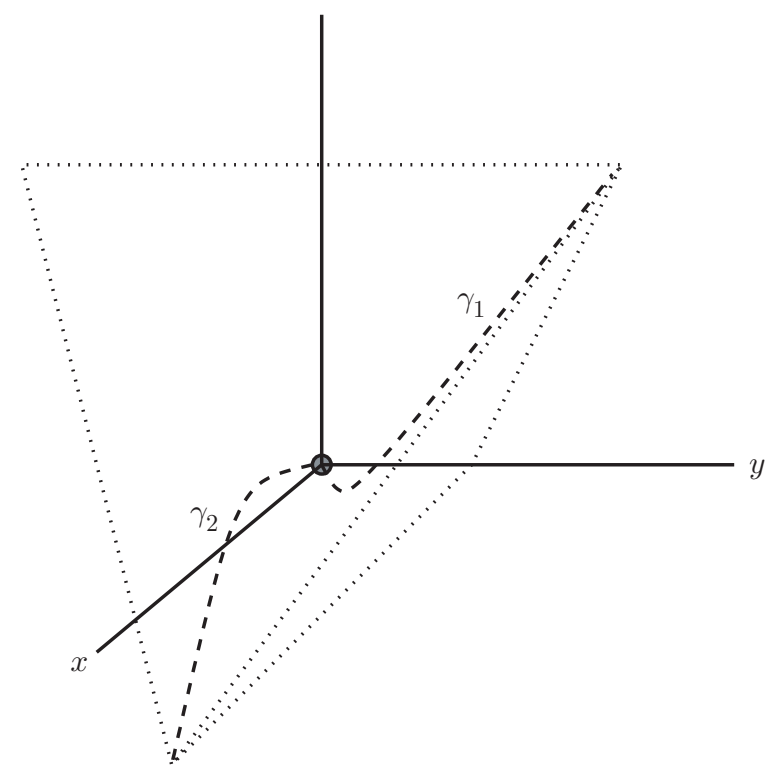

Figure 7. Two intersecting strips with four point generating set.

In light of Corollary 2, the crucial thing to check is that these curves can be constructed with non-vanishing curvature. A more complete list of requirements is as follows:

(1) The curves coincide with the sphere near the origin.

(2) The curvatures of the curves never vanish.

(3) All points on the curves, except the vertices, lie interior to the tetrahedron $T$.

(4) The tangent-binormal plane is transverse to the three faces at each vertex.

We first construct $\gamma_{1}$ parameterized as a function of $y$.

$$
\gamma_{1}(y)=(x(y), y, z(y)) .
$$

For some $\rho<r$, we require $x(y)=y$ and $z(y)=-r+\sqrt{r^{2}-2 y^{2}}$ for $y<\rho$. Notice that $z^{\prime \prime}(0)=-2 / r$ can be made arbitrarily large in magnitude by choosing $r$ (and $\rho)$ small.

We extend $z$ to be even and smooth as shown in Figure 8(a) with $z^{\prime}(y)=0$ for exactly three points $y=0, \pm \epsilon$, and $z^{\prime \prime}(y)=0$ for exactly two points $y= \pm \delta$ with $\rho<\delta<\epsilon$.

Note also that $z<2 y-1$ for $|y|<1$ in accordance with condition (3), and $0<z^{\prime}(1)<2$ in accordance with condition (4).

We extend $x$ to be odd and smooth as shown in Figure 8(b) with exactly four critical points at $\pm \epsilon$ and $\pm a$, and $x^{\prime \prime}(y)=0$ at exactly two points $y= \pm b$ outside of $[-\rho, \rho]$ with $\epsilon<|b|$. Observe that $x$ is linear on $[-\rho, \rho]$. It is also clear that $x=x(y)$ may be chosen with arbitrarily small magnitude (sup-norm) and with $x^{\prime}(1)$ arbitrarily close to zero. In this way, conditions 3 and 4 will not be violated by $\gamma_{1}$. 


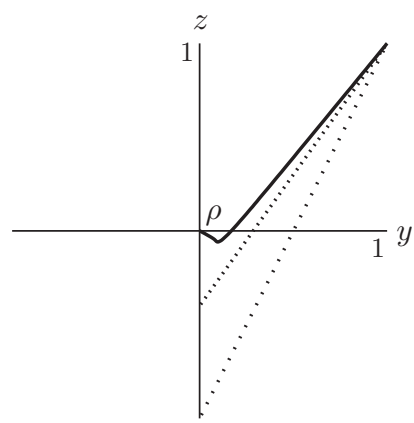

(a)

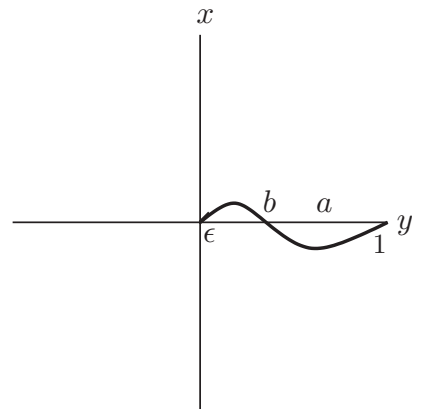

(b)

Figure 8. Constructing $\gamma_{1}$; (a) $z(y)$, (b) $x(y)$.

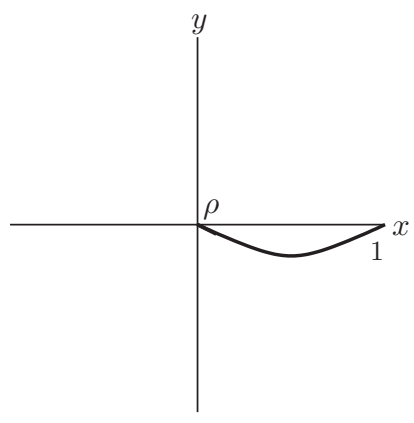

(a)

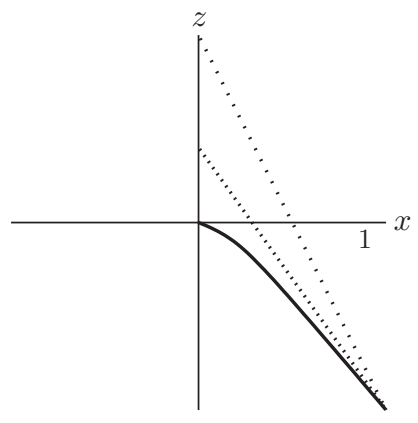

(b)

Figure 9. Constructing $\gamma_{2}$; (a) $y(y)$, (b) $z(y)$.

It remains to be shown that $x$ and $z$ can be chosen so that the resulting curve $\gamma_{1}:[0,1] \rightarrow T \subset \mathbb{R}^{3}$ has non-vanishing curvature. Denoting derivatives with respect to $y$ by primes and derivatives with respect to arclength by dots, we find $\ddot{\gamma}_{1}$ is proportional to

$$
\left(x^{\prime \prime}, 0, z^{\prime \prime}\right)\left(1+x^{\prime 2}+z^{\prime 2}\right)-\left(x^{\prime}, 1, z^{\prime}\right)\left(x^{\prime} x^{\prime \prime}+z^{\prime} z^{\prime \prime}\right) .
$$

The second component vanishes only if $x^{\prime} x^{\prime \prime}+z^{\prime} z^{\prime \prime}=0$. From this it follows that the first and third components vanish only if $x^{\prime \prime}=z^{\prime \prime}=0$. Since $z^{\prime \prime}(y) \neq 0$ for $|y| \leq \rho, x^{\prime \prime}(y) \neq 0$ for $\rho<|y| \leq \epsilon$, and $z^{\prime \prime}(y) \neq 0$ for $|y|>\epsilon$, we see that the curvature never vanishes.

The curve $\gamma_{2}(x)=(x, y(x), z(x))$ is chosen similarly according to Figure 9, In this case, the graph of $z$ may be chosen inflection free. Again, $y$ is linear on $[-\rho, \rho]$ and $z$ is prescribed on the same interval by the requirement that the curve lies on the spherical junction.

The construction is completed by choosing $r$ small enough according to the proof of Corollary 2, It is a technicality to check that $\gamma_{1}$ and $\gamma_{2}$ can be chosen with curvatures bounded away from zero as $r \rightarrow 0$, so that the first term on the right of equation (77) tends to $+\infty$.

As a final note, it is clear that the method of construction of the last example can be used to construct extremely complicated embedded disks $\mathcal{S}$ of positive curvature. 
For example, if $\mathcal{N}$ is any simply connected network of transversally intersecting smooth curves and $\epsilon>0$, then there is a positively curved embedded disk $\mathcal{S}$ that enters the $\epsilon$-neighborhood of every point in $\mathcal{N}$. If the curves in the network, are arranged to have isolated endpoints $q_{1}, \ldots, q_{k}$ on the vertices of a convex polyhedron $T$ with all other points of the network in the interior of $T$, then it can be arranged that $\mathcal{S}$ has generating set $q_{1}, \ldots, q_{k}$.

\section{REFERENCES}

1. S. Alexander and M. Ghomi, The convex hull property and topology of hypersurfaces with nonnegative curvature, Adv. Math. 180 (2003), 324-354. MR.2019227

2. M. do Carmo and E. Lima, Immersions of manifolds with non-negative sectional curvatures, Bol. Soc. Brasil Mat. 2 (1971), 9-22. MR0328828 (48:7170)

3. M. Ghomi, Strictly convex submanifolds and hypersurfaces of positive curvature, J. Differential Geom. 57 (2001), 239-271. MF 1879227 (2002k:52001)

4. J. Hadamard, Sur certaines proprietés des trajectoires en dynamique, J. Math. Pures Appl. 3 (1897), 331-387.

5. S.-T. Yau, Open Problems in Geometry, Proc. of Symp. in Pure Mathematics, vol. 54, Part I, 1993. MR1216573 (94k:53001)

School of Mathematics, Georgia Institute of Technology, Atlanta, Georgia 30332

E-mail address: mccuan@math.gatech.edu 